\title{
Peak emission altitude of Saturn's $\mathrm{H}_{3}^{+}$aurora
}

\author{
Tom S. Stallard, ${ }^{1}$ Henrik Melin, ${ }^{1}$ Steve Miller, ${ }^{2}$ Sarah V. Badman, ${ }^{3}$ Robert H. Brown, ${ }^{4}$ \\ and Kevin H. Baines ${ }^{5}$ \\ Received 20 June 2012; revised 2 July 2012; accepted 2 July 2012; published 7 August 2012.
}

[1] Here we present the first detailed measurement of the altitudinal profile of $\mathrm{H}_{3}^{+}$emission within Saturn's ionosphere, made using images taken by the VIMS instrument on Cassini on 11-12 October 2006, during a chance alignment between the visible limb of the planet and the position of the main auroral emission. Using this, we show that the emission profile of $\mathrm{H}_{3}^{+}$can be fitted to a reasonable accuracy with a Gaussian, producing a calculated peak emission altitude at $1155( \pm 25) \mathrm{km}$ that differs significantly from previous observations of the UV emission profile, and also from the predictions of models that calculated the $\mathrm{H}_{3}^{+}$emission profile, which suggested that there would be extended emission above the peak emission altitude. This lack of extended emission is most simply explained by differences in the scale height of $\mathrm{H}$ and $\mathrm{H}_{2}$, suggesting that models overestimate $\mathrm{H}_{2}$ at high altitudes, with little $\mathrm{H}_{2}$ from $2000 \mathrm{~km}$ above the 1 bar level. Citation: Stallard, T. S., H. Melin, S. Miller, S. V. Badman, R. H. Brown, and K. H. Baines (2012), Peak emission altitude of Saturn's H $\mathrm{H}_{3}^{+}$aurora, Geophys. Res. Lett., 39, L15103, doi:10.1029/ 2012GL052806.

\section{Introduction}

[2] Our understanding of the main auroral oval at Saturn has increased greatly in recent years, following a number of important investigations using both ultraviolet (UV) and infrared (IR) instruments on the Cassini spacecraft in orbit around Saturn, and through UV imaging from the Hubble Space Telescope (HST). The main auroral oval is strongly controlled by the solar wind and is generally thought to be formed by upward-directed field-aligned currents associated with the open-closed field line boundary [Bunce et al., 2008].

[3] These currents direct energetic charged particles into the upper atmosphere of Saturn, both exciting and ionising $\mathrm{H}$ and $\mathrm{H}_{2}$. This results in prompt UV emission from both $\mathrm{H}$ Lyman- $\alpha$ and $\mathrm{H}_{2}$ Lyman and Werner bands (typically observed by HST through broad filters that cannot differentiate these emissions, so that we broadly describe them

\footnotetext{
${ }^{1}$ Radio and Space Plasma Physics Group, Department of Physics and Astronomy, University of Leicester, Leicester, UK.

${ }^{2}$ Atmospheric Physics Laboratory, Department of Physics and Astronomy, University College London, London, UK.

${ }^{3}$ Institute of Space and Astronautical Science, Japan Aerospace Exploration Agency, Sagamihara, Japan.

${ }^{4}$ Lunar and Planetary Laboratory and Steward Observatory, University of Arizona, Tucson, Arizona, USA.

${ }^{5}$ SSEC, University of Wisconsin-Madison, Madison, Wisconsin, USA.

Corresponding author: T. S. Stallard, Radio and Space Plasma Physics Group, Department of Physics and Astronomy, University of Leicester, University Road, Leicester LE1 7RH, UK. (tss@ion.le.ac.uk)

C2012. American Geophysical Union. All Rights Reserved. 0094-8276/12/2012GL052806
}

here as UV emission). The ionisation of $\mathrm{H}_{2}$ results in the formation of $\mathrm{H}_{2}^{+}$. This $\mathrm{H}_{2}^{+}$then reacts with further $\mathrm{H}_{2}$ to form $\mathrm{H}_{3}^{+}$, which is, above the homopause, a relatively stable ion lasting approximately 10 minutes [Melin et al., 2011]. As a result of this stability, the $\mathrm{H}_{3}^{+}$becomes (quasi-) thermalized with the surrounding neutral atmosphere, resulting in infrared ro-vibrational emission, mainly in the 3-5 micron wavelength region [Miller et al., 1990].

[4] During periods of solar wind rarefaction, the main UV auroral emission forms an oval positioned at $\sim 74$ degrees latitude (all latitudes in this paper are in planetocentric coordinates), though the specific location of the oval can vary by as much as \pm 3 degrees [Badman et al., 2006]. Both statistical [Badman et al., 2011] and simultaneous [Melin et al., 2011] observations of the $\mathrm{H}_{3}^{+}$aurora suggest that it is typically co-located with the main UV emission. During periods of compression in the solar wind, both $\mathrm{H}_{3}^{+}$ and UV aurora change in morphology, leading firstly to dawn brightening and then, with increasing solar wind pressure, to the formation of a main auroral spiral [Grodent et al., 2005; Stallard et al., 2008]. Large compressions in the solar wind cause a dramatic change in the auroral morphology as the entire dawn side of the polar region is filled with bright emission [Grodent et al., 2005; Stallard et al., 2007].

[5] Spectral analysis and modelling of the UV $\mathrm{H}_{2}$ aurora show that it is typically formed by precipitating electrons with an energy of $\sim 0.1-5 \mathrm{keV}$ [Melin et al., 2011; Gérard et al., 2009; Gustin et al., 2009]. Gérard et al. [2009] have studied the emission altitude of the UV main aurora, utilising a collection of HST images. Their study results in a range of peak emission altitudes between $800-1200 \mathrm{~km}$, with an average peak emission altitude of $1145 \pm 305 \mathrm{~km}$. This result is supported by measurements of the UV color ratio which typically show negligible levels of absorption by methane [Gérard et al., 2009]. Gérard et al.'s peak emission altitude is well above the modeled homopause $(\sim 500$ $800 \mathrm{~km}$ ) [Moses et al., 2000]. This means that Saturn differs significantly from Jupiter, where the peak UV emission comes from well below the homopause [Grodent and Gérard, 2001]. Thus the UV emission from Saturn may be co-located in altitude with the IR $\mathrm{H}_{3}^{+}$emission, while this is not the case for Jupiter, as any $\mathrm{H}_{3}^{+}$produced below the homopause is quickly destroyed through its reaction with the hydrocarbons there [Achilleos et al., 1998].

[6] To date, however, the altitude of $\mathrm{H}_{3}^{+}$auroral emission has not actually been measured, although model intensity profiles have been generated [Tao and Fujimoto, 2011; Miller et al., work in progress]. While $\mathrm{H}_{3}^{+}$occurs at all latitudes in Saturn's ionosphere, it is largely confined to the high-latitude/auroral regions [Stallard et al., 2012], where it is the product of the same energetic particle precipitation that 
Table 1. Values for Each Image Shown in Figure $1^{\text {a }}$

\begin{tabular}{|c|c|c|c|c|c|c|c|c|c|c|}
\hline Image & DOY & $\begin{array}{l}\text { Time } \\
\text { (h:m) }\end{array}$ & Itime (s) & $\begin{array}{l}\text { Oval Latitude } \\
\text { (deg) }\end{array}$ & $\begin{array}{l}\text { Limb Latitude } \\
\text { (deg) }\end{array}$ & $\begin{array}{l}\Delta \text { Latitude } \\
\text { (deg) }\end{array}$ & $\begin{array}{l}\text { Sub-SC Latitude } \\
\text { (deg) }\end{array}$ & $\begin{array}{l}\text { SC Dist. } \\
\quad\left(\mathrm{R}_{\mathrm{S}}\right)\end{array}$ & $\begin{array}{l}\text { Peak Height } \\
(\mathrm{km})\end{array}$ & $\begin{array}{l}\text { FWHM } \\
(\mathrm{km})\end{array}$ \\
\hline $1^{\mathrm{b}}$ & $2008-284$ & $22: 39$ & 1000 & -76.0 & -74.0 & 2.0 & -23.9 & 5.52 & $1023 \pm 8$ & $1039 \pm 11$ \\
\hline 2 & $2008-284$ & $23: 53$ & 80 & -76.7 & -75.9 & 0.8 & -20.5 & 5.54 & $1157 \pm 17$ & $714 \pm 21$ \\
\hline 3 & $2008-284$ & $23: 59$ & 80 & -77.1 & -76.5 & 0.6 & -20.0 & 5.54 & $1138 \pm 18$ & $702 \pm 23$ \\
\hline 4 & $2008-285$ & $00: 05$ & 80 & -77.2 & -76.9 & 0.3 & -19.5 & 5.55 & $1148 \pm 14$ & $716 \pm 18$ \\
\hline 5 & $2008-285$ & $00: 18$ & 80 & -77.4 & -78.0 & 0.6 & -18.4 & 5.56 & $1166 \pm 16$ & $657 \pm 18$ \\
\hline 6 & $2008-285$ & $00: 24$ & 160 & -77.5 & -78.9 & 1.4 & -17.7 & 5.57 & $1161 \pm 7$ & $652 \pm 8$ \\
\hline 7 & $2008-285$ & $00: 39$ & 160 & -78.0 & -80.0 & 2.0 & -16.4 & 5.60 & $1109 \pm 8$ & $695 \pm 9$ \\
\hline 8 & $2008-285$ & $00: 55$ & 160 & -78.4 & -81.6 & 3.2 & -15.0 & 5.62 & $1038 \pm 7$ & $660 \pm 8$ \\
\hline 9 & $2008-285$ & $01: 12$ & 160 & -79.2 & -83.2 & 4.0 & -13.6 & 5.66 & $990 \pm 6$ & $621 \pm 7$ \\
\hline
\end{tabular}

${ }^{\text {a }}$ The table consists of image number (Image); the day of year in which the image was taken (DOY); the integration time per pixel for each image (Itime); the calculated latitude of the main auroral oval on the body of the planet (Oval Latitude); the mean latitude at the limb (Limb Latitude); the difference between the calculated main oval latitude and the latitude on the limb, a proxy for the alignment between the main oval and the limb ( $\Delta$ Latitude); the mean sub-spacecraft latitude during the observation (Sub-SC Latitude); the mean spacecraft distance from Saturn (SC Dist.); the calculated altitude of the peak $\mathrm{H}_{3}^{+}$emission (Peak Height); and the altitudinal full-width half-maximum of the Gaussian fitted to the $\mathrm{H}_{3}^{+}$emission profile (FWHM).

${ }^{\mathrm{b}}$ Image 1 does not have a clear view of the aurora on the body of the planet, so that the measured latitude may be less accurate. It was also taken over a much longer period of time and the high FWHM suggests the oval has changed in apparent peak height over the period of the observation, as the aurora moved into better alignment.

results in the UV aurora. Local $\mathrm{H}_{3}^{+}$emission intensities reflect both the ion density and temperature, since emission per ion increases with temperature [Miller et al., 2010]. The column averaged temperature of Saturn's auroral $\mathrm{H}_{3}^{+}$emission, as derived from IR observations, lies in the $\sim 350$ $500 \mathrm{~K}$ range [Melin et al., 2007, 2011]. In some models, however, the temperature is predicted to reach as high as $800 \mathrm{~K}$ at the top of the auroral thermosphere [e.g., MüllerWodarg et al., 2006], resulting in a factor of $\sim 100$ increase in the $\mathrm{H}_{3}^{+}$emission per molecule. As a result of this, models of the IR intensity profile show extended emission at higher altitudes relative to the UV, although it is not thought that this will strongly affect the peak emission altitude [Tao and Fujimoto, 2011].

\section{Main Emission Altitude}

[7] Reliable measurements of the altitudinal emission profile in the auroral oval require a relatively rare alignment, with the aurora located directly on the limb in the line-ofsight. Such an alignment cannot be pre-planned. Here we present observations made by the VIMS instrument on Cassini [Brown et al., 2004], made during a chance alignment in 2006, on days 284 (11 October) and 285 (12 October); these are listed in Table 1.

[8] Unfortunately, there are no concurrent measurements of solar wind conditions during this period. A VIMS observation taken at 02:38 on day 284 [Stallard et al., 2008, Figure 2] shows a spiral morphology, however, suggesting that there was a moderate solar wind compression within two Saturnian days of the observations presented here [Cowley et al., 2005].

[9] From the VIMS images taken at various times during Days 284 and 285, we have produced nine composite images consisting of emission from multiple $\mathrm{H}_{3}^{+}$lines (see Figure 1). The composite images made use of individual VIMS measurements centred on wavelengths of 3.4175, 3.5319, 3.6159, 3.6672, 4.2004 and $4.3529 \mu \mathrm{m}$. Each of these had effective wavelength coverage modelled as a Gaussian with a full-width at half-maxima (FWHM) of 0.0180, 0.0178, $0.0195,0.0204,0.0241$ and $0.0231 \mu \mathrm{m}$, respectively. There is a significant contribution from sunlight reflected from the planet as high as $500 \mathrm{~km}$ above the limb. So, from this sequence of images, we respectively subtracted background images centred on a wavelength of $3.3676,3.5645$, $3.6329,3.7015,4.1850$ and $4.3167 \mu \mathrm{m}$, with FWHMs of $0.0180,0.01828,0.01980,0.02077,0.02414$ and $0.0233 \mu \mathrm{m}$ respectively, and multiplied by an empirically defined factor of $0.45,0.7,0.7,0.7,0.75$ and 0.8 to account for wavelength variations in the reflected sunlight brightness. Unfortunately we were not able to use images containing emission from the Q-branch $\mathrm{H}_{3}^{+}$lines, which lie between 3.95-4.05 $\mu \mathrm{m}$, since background subtraction proved unfeasible. After background subtraction, each individual wavelength image was cleaned to remove instrumental errors. These background-subtracted, cleaned images were then combined to produce the images shown in Figure 1. These processed images were then used to determine the location of the auroral "limb" emission on the planet.

[10] The processed images were initially mapped against a raised planetary 'surface', calculated using a 1-bar surface taken from the NASA SPICE toolkit, in turn defined from the values in Seidelmann et al. [2007] and raised by $1000 \mathrm{~km}$ orthogonal to the surface of the oblate spheroid; this raised surface is a first approximation of the location of the $\mathrm{H}_{3}^{+}$location based upon estimates for the UV peak altitude [Gérard et al., 2009]. This mapping is used in order to compare the latitudes of the aurora and the limb. The location of the limb was calculated from this raised surface by averaging the latitude provided by the NASA SPICE toolkit along the limb within these images. The location of the main oval was measured by binning the intensity of each image into one degree latitude bins, ignoring any data within $800 \mathrm{~km}$ of the limb (to avoid line-of-sight enhancements at the edge of the planet). This was fitted, for the sake of simplicity, using a Gaussian with a quadratic background, in order to find the latitude of the peak intensity of the main auroral emission, resulting in an effective auroral emission latitude for comparison with the limb latitude. These values, shown in Table 1, indicate that the difference between the effective auroral latitude and the limb latitude ranged from $0.3^{\circ}$ (Image 4 ) to $4.0^{\circ}$ (Image 9), reflecting the changing line-of-sight between the spacecraft, the limb and the auroral oval.

[11] The processed images were then mapped to a planetary 'surface' at the 1 bar level (as shown in Figure 1), again 


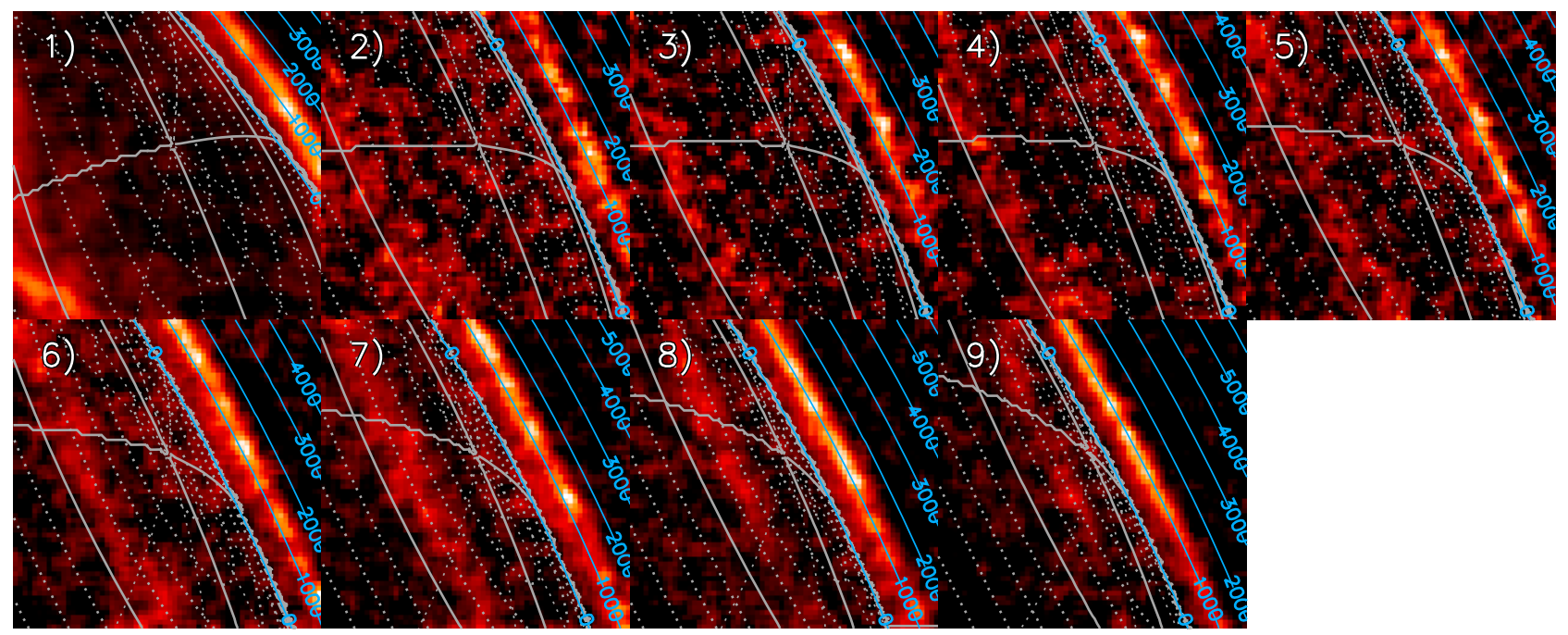

Figure 1. (a-g) A sequence of Cassini-VIMS images, from 11-12 October 2006 (days 284-285), of $\mathrm{H}_{3}^{+}$emission from the southern aurora region, the details for which are given in Table 1. Overlain on these images are the planetary coordinates at the 1 bar level. Latitude is shown in minor steps of two degrees (dots) and major steps of ten degrees (three-dot dashes). Local time is given in minor steps of two hours (dots) and major steps of six hours (three-dot dashes), with noon to the left and midnight over the limb for each image. Height above the 1-bar limb is also shown, in steps of $1000 \mathrm{~km}$ (blue). The segment of the main auroral oval that lies on, or close to, the limb of the planet shows up as a bright arc on the right-hand side of the images. Towards the left, less intense auroral oval emission can be seen against the body of the planet. It is the auroral emission above the limb of the planet that is used to produce altitude profiles reported in this paper.

taken from the NASA SPICE toolkit. The distance in the plane of view, orthogonal to the limb, was then calculated, above or below the limb, for every pixel. Using this, the intensity of those pixels above the limb were then binned into an altitudinal histogram consisting of $50 \mathrm{~km}$ bins, with unfilled bins being ignored in the resultant profile. The resultant histograms were then fitted with a Gaussian with a quadratic background, in order to produce a value for the peak emission height, also shown in Table 1.

[12] The sub-spacecraft latitude changes through the period of Days 284-285, so that the observed limb of the planet shifts to higher latitudes as time goes on. Fortuitously, however, the latitude of the main auroral oval also appears to be moving poleward, coincidentally maintaining a limb alignment with Cassini. (This may reflect changes in the solar wind strength, following the compression noted previously at the start of Day 284.) Nonetheless, as noted previously, the difference between the effective auroral emission latitude and the limb latitude does change as a function of time. Table 1 shows that this difference drops monotonically from $2.0^{\circ}$ in Image 1 to $0.3^{\circ}$ in Image 4, before increasing (again monotonically) to $4.0^{\circ}$ in Image 9 . Given the viewing geometry of the spacecraft, the calculated altitude of peak $\mathrm{H}_{3}^{+}$emission will tend to decrease as the difference between the two latitudes increases. Table 1 tends to confirm this, although image-to-image divergences from this trend occur as a result both of inaccuracies in our fitting techniques and potential real-time variations in the aurorae themselves.

[13] In order to produce as accurate an emission profile as possible, we have therefore selected only those images for which the difference between limb and auroral latitude is $<1.5^{\circ}$, i.e. images $2-5$. This produces an emission profile with a signal-to-noise ratio increased with respect to single images, shown in Figure 2. At lower altitudes, below $500 \mathrm{~km}$ above the 1-bar level, we see an emission intensity around $20 \%$ of the maximum. This emission may be composed of genuine low-altitude emission, but line-of-sight effects through the polar cap means that Cassini is also viewing some of the weaker emission at higher latitudes. Above

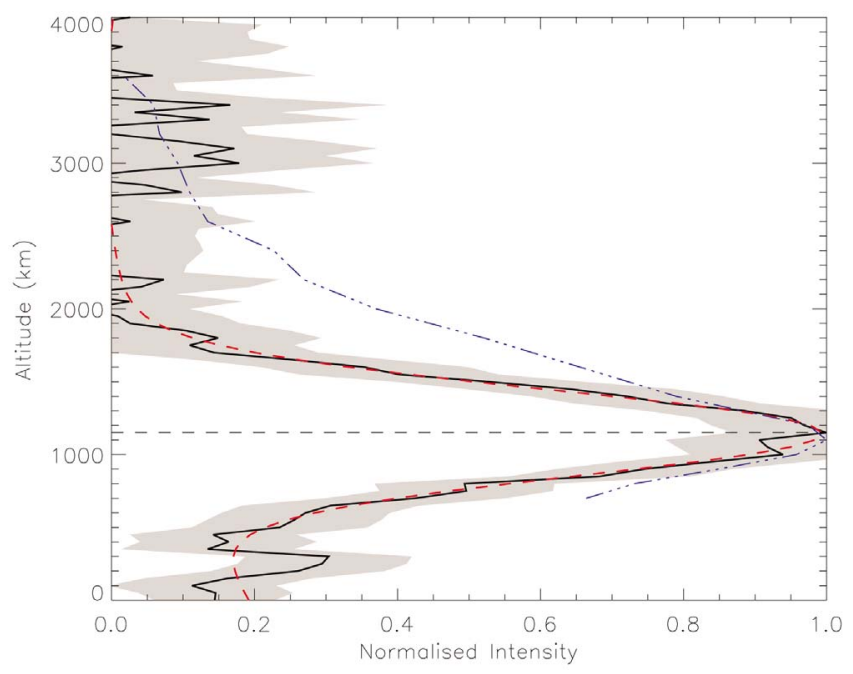

Figure 2. Altitudinal profile of normalised $\mathrm{H}_{3}^{+}$auroral intensity derived from a combination of Images $2-5$. The intensity profile is a coadded histogram in $50 \mathrm{~km}$ bins with altitude (black solid line), using images where the latitude at the limb is within 1.5 degrees of the measured auroral oval location. This profile was fitted with a Gaussian with a quadratic background (red dashed line) in order to measure the peak emission altitude (vertical dashed line). Also shown, for comparison, is the UV intensity profile measured by Gérard et al. [2009] (three-dot dash blue line). 
$500 \mathrm{~km}$, however, the intensity increases rapidly up to 1100 $1200 \mathrm{~km}$, before declining rapidly to zero at around $2000 \mathrm{~km}$ above the 1-bar level. The emission peak is well fitted by a simple Gaussian, combined with a quadratic background; we calculate a peak altitude of $1155( \pm 16) \mathrm{km}$ and a full-width half-maximum of $683( \pm 19) \mathrm{km}$ (all errors are calculated by measuring the background noise and iteratively modeling the standard error that this noise produces in Gaussian fits to the respective modeled auroral profiles).

[14] Given the relative accuracy of the fitting of this data, the changes in altitude measured in the each observation may represent true variability within the auroral region. The measured peak heights of UV aurora vary significantly with time, with direct measurements varying between 800 $1200 \mathrm{~km}$, so it is possible that the change in altitude is driven entirely by actual changes in the peak emission, in turn caused by variations in the energy distribution of precipitating particles changing the mean depth of penetration. However, the narrowing of the auroral oval over the period of these observations suggests a strengthening compression in the solar wind, and is not matched by a deepening auroral peak with time. In addition, past observations have shown that the aurora is not always at the same colatitude at different local times, so that our suggested auroral limb alignment is merely an estimation of the true alignment of the main emission on the limb, and variations within the dataset may be entirely due to mis-alignment; in the case that the peak altitude was fixed through this event, then the peak altitude would be best measured as $=>1166 \mathrm{~km}$, the highest measured peak altitude in this alignment event.

[15] In Figure 2, we also overplot the best emission profile derived for the UV by Gérard et al. [2009]; both profiles are line-of-sight measurements and can be directly compared. The peak occurs at more or less the same altitude in both the UV and IR, within the range of errors, strongly suggesting that the maximum emission is produced by the same energetic particles, as has been previously suggested by both modelling and analysis of UV spectra of the main auroral emission [Tao and Fujimoto, 2011; Melin et al., 2011].

[16] However, the UV profile cannot be fitted with a simple Gaussian and extends to a higher altitude than the $\mathrm{H}_{3}^{+}$. This contradicts the previously modeled relative intensity of auroral volume emission [Tao and Fujimoto, 2011], which has a bulge of higher altitude emission in the IR, relative to the UV. The simplest explanation for the difference between the two profiles is that it is caused by a chemical difference in the production of the aurora.

[17] As discussed earlier, $\mathrm{H}_{3}^{+}$is produced almost entirely from $\mathrm{H}_{2}$, while the Gérard et al. [2009] UV profile shown in Figure 2 was produced from a HST image using the F115 filter, which includes emission from both the H Lyman- $\alpha$ line and $\mathrm{H}_{2}$ Lyman and Werner bands. Simple scale-height considerations $\left(\mathrm{H}_{\mathrm{a}}=\left(\mathrm{kT} / \mathrm{m}_{\mathrm{a}} \mathrm{g}\right)^{1 / 2}\right)$ mean that the population of $\mathrm{H}$ extends to much higher altitudes in Saturn's ionosphere than that of $\mathrm{H}_{2}$ (and, consequently, $\mathrm{H}_{3}^{+}$). So this result may be providing important contextual information about the mixing ratios of $\mathrm{H}$ and $\mathrm{H}_{2}$ in the upper atmosphere. It implies, for example, that at higher altitudes, the UV emission comes uniquely from H Lyman- $\alpha$ emission (presumably excited by energetic electrons or, possibly, through resonant scattering from solar $\mathrm{H}$ Lyman- $\alpha$ ), suggesting that there is little $\mathrm{H}_{2}$ more than $2000 \mathrm{~km}$ above the 1 bar level.
Given that most model constraints come from equatorial measurements [e.g., Majeed et al., 1991], this suggests neutral conditions within the auroral regions are different from those used in past models. A more detailed understanding of the comparative intensity of the IR and UV profiles, and the relative molecular populations that this provides will, however, require simultaneous observations of both wavelengths above the limb.

[18] Acknowledgments. This work was supported by a RCUK Fellowship for T.S. and by the UK STFC for H.M. and T.S.

[19] The Editor thanks Darrell Strobel and an anonymous reviewer for their assistance in evaluating this paper.

\section{References}

Achilleos, N., S. Miller, J. Tennyson, A. D. Aylward, I. Mueller-Wodarg, and D. Rees (1998), JIM: A time-dependent, three-dimensional model of Jupiter's thermosphere and ionosphere, J. Geophys. Res., 103, 20,089-20,112, doi:10.1029/98JE00947.

Badman, S. V., S. W. H. Cowley, J.-C. Gérard, and D. Grodent (2006), A statistical analysis of the location and width of Saturn's southern auroras, Ann. Geophys., 24, 3533-3545, doi:10.5194/angeo-24-3533-2006.

Badman, S. V., N. Achilleos, K. H. Baines, R. H. Brown, E. J. Bunce, M. K. Dougherty, H. Melin, J. D. Nichols, and T. Stallard (2011), Location of Saturn's northern infrared aurora determined from Cassini VIMS images, Geophys. Res. Lett., 38, L03102, doi:10.1029/2010GL046193.

Brown, R. H., et al. (2004), The Cassini Visual And Infrared Mapping Spectrometer (VIMS) investigation, Space Sci. Rev., 115, 111-168, doi:10.1007/s11214-004-1453-X.

Bunce, E. J., et al. (2008), Origin of Saturn's aurora: Simultaneous observations by Cassini and the Hubble Space Telescope. J. Geophys. Res. 113, A09209, doi:10.1029/2008JA013257.

Cowley, S. W. H., S. V. Badman, E. J. Bunce, J. T. Clarke, J.-C. Gérard, D. Grodent, C. M. Jackman, S. E. Milan, and T. K. Yeoman (2005), Reconnection in a rotation-dominated magnetosphere and its relation to Saturn's auroral dynamics, J. Geophys. Res., 110, A02201, doi:10.1029/ 2004JA010796.

Gérard, J.-C., B. Bonfond, J. Gustin, D. Grodent, J. T. Clarke, D. Bisikalo, and V. Shematovich (2009), Altitude of Saturn's aurora and its implications for the characteristic energy of precipitated electrons, Geophys. Res. Lett., 36, L02202, doi:10.1029/2008GL036554.

Grodent, D., and J.-C. Gérard (2001), A self-consistent model of the Jovian auroral thermal structure, J. Geophys. Res., 106, 12,933-12,952, doi:10.1029/2000JA900129.

Grodent, D., J.-C. Gérard, S. W. H. Cowley, E. J. Bunce, and J. T. Clarke (2005), Variable morphology of Saturn's southern ultraviolet aurora, J. Geophys. Res., 110, A07215, doi:10.1029/2004JA010983.

Gustin, J., J.-C. Gérard, W. Pryor, P. D. Feldman, D. Grodent, and G. Holsclaw (2009), Characteristics of Saturn's polar atmosphere and auroral electrons derived from HST/STIS, FUSE and Cassini/UVIS spectra, Icarus, 200, 176-187, doi:10.1016/j.icarus.2008.11.013.

Majeed, T., R. V. Yelle, and J. C. McConnell (1991), Vibrationally excited $\mathrm{H}_{2}$ in the outer planets thermosphere: Fluorescence in the Lyman and Werner bands, Planet. Space Sci., 39, 1591-1606, doi:10.1016/00320633(91)90085-O.

Melin, H., S. Miller, T. Stallard, L. M. Trafton, and T. R. Geballe (2007), Variability in the $\mathrm{H}_{3}^{+}$emission of Saturn: Consequences for ionisation rates and temperature, Icarus, 186, 234-241, doi:10.1016/j.icarus.2006.08.014.

Melin, H., T. Stallard, S. Miller, J. Gustin, M. Galand, S. V. Badman, W. R. Pryor, J. O’Donoghue, R. H. Brown, and K. H. Baines (2011), Simultaneous Cassini VIMS and UVIS observations of Saturn's southern aurora: Comparing emissions from $\mathrm{H}, \mathrm{H}_{2}$ and $\mathrm{H}_{3}^{+}$at a high spatial resolution, Geophys. Res. Lett., 38, L15203, doi:10.1029/2011GL048457.

Miller, S., R. D. Joseph, and J. Tennyson (1990), Infrared emissions of $\mathrm{H}_{3}^{+}$ in the atmosphere of Jupiter in the 2.1 and 4.0 micron region, Astrophys. $J ., 360$, L55-L58, doi:10.1086/185811.

Miller, S., T. Stallard, H. Melin, and J. Tennyson (2010), $\mathrm{H}_{3}^{+}$cooling in planetary atmospheres, Faraday Discuss., 147, 283-291, doi:10.1039/ c004152c

Moses, J. I., B. Bézard, E. Lellouch, G. R. Gladstone, H. Feuchtgruber, and M. Allen (2000), Photochemistry of Saturn's atmosphere. I. Hydrocarbon chemistry and comparisons with ISO observations, Icarus, 143, 244-298, doi:10.1006/icar.1999.6270.

Müller-Wodarg, I. C. F., M. Mendillo, R. V. Yelle, and A. D. Aylward (2006), A global circulation model of Saturn's thermosphere, Icarus, 180, 147-160, doi:10.1016/j.icarus.2005.09.002. 
Seidelmann, P. K., et al. (2007), Report of the IAU/IAG Working Group on cartographic coordinates and rotational elements: 2006, Celestial Mech. Dyn. Astron., 98, 155-180, doi:10.1007/s10569-007-9072-y.

Stallard, T., S. Miller, H. Melin, M. Lystrup, M. Dougherty, and N. Achilleos (2007), Saturn's auroral/polar $\mathrm{H}_{3}^{+}$infrared emission. I. General morphology and ion velocity structure, Icarus, 189, 1-13, doi:10.1016/j. icarus.2006.12.027.

Stallard, T., S. Miller, H. Melin, M. Lystrup, S. W. H. Cowley, E. J. Bunce, N. Achilleos, and M. Dougherty (2008), Jovian-like aurorae on Saturn, Nature, 453, 1083-1085, doi:10.1038/nature07077.
Stallard, T. S., H. Melin, S. Miller, J. O’Donoghue, S. W. H. Cowley, S. V. Badman, A. Adriani, R. H. Brown, and K. H. Baines (2012), Temperature changes and energy inputs in Giant Planet atmospheres: what we are learning from $\mathrm{H}_{3}^{+}$, Philos. Trans. R. Soc. A, in press.

Tao, C. S. V. B., and M. Fujimoto (2011), UV and IR auroral emission model for the outer planets: Jupiter and Saturn comparison, Icarus, 213, 581-592, doi:10.1016/j.icarus.2011.04.001. 